\title{
ANALYSIS AND REDUCTION OF REFERENCE FRAMES FOR MOTION ESTIMATION IN MPEG-4 AVC/JVT/H.264
}

\author{
Yu-Wen Huang ${ }^{1, *}$, Bing-Yu Hsieh ${ }^{1}$, Tu-Chih Wang ${ }^{1}$, Shao-Yi Chien ${ }^{1}$, \\ Shyh-Yih $\mathrm{Ma}^{2}$, Chun-Fu Shen ${ }^{2}$, and Liang-Gee Chen ${ }^{1}$ \\ 1. DSP/IC Design Lab., Graduate Institute of Electronics Engineering and \\ Department of Electrical Engineering, National Taiwan University, yuwen@ video.ee.ntu.edu.tw \\ 2. Vivotek Incorporation, steve@vivotek.com
}

\begin{abstract}
In the new video coding standard, MPEG-4 AVC/JVT/H.264, motion estimation is allowed to use multiple reference frames. The reference software adopts full search scheme, and the increased computation is in proportion to the number of searched reference frames. However, the reduction of prediction residues is highly dependent on the nature of sequences, not on the number of searched frames. In this paper, we present a method to speed up the matching process for multiple reference frames. For each macroblock, we analyze the available information after intra prediction and motion estimation from previous one frame to determine whether it is necessary to search more frames. The information we use includes selected mode, inter prediction residues, intra prediction residues, and motion vectors. Simulation results show that the proposed algorithm can save up to $90 \%$ of unnecessary frames while keeping the average miss rate of optimal frames less than $4 \%$.
\end{abstract}

\section{INTRODUCTION}

Joint Video Team (JVT) gathered experts from ISO/IEC MPEG-4 Advanced Video Coding (AVC) and ITU-T H.264 to develop the latest standard. The new standard significantly outperforms previous ones in bit-rate reduction. Compared to MPEG-4 advanced simple profile, up to $50 \%$ of bit-rate reduction can be achieved. Such improvement mainly comes from the prediction part [1]. Motion estimation at quarter-pixel accuracy with variable block sizes and multiple reference frames greatly reduces prediction errors. Even if inter-frame prediction cannot find a good match, intra prediction will make it up instead of directly coding the texture.

The reference software, JM4.3 [2], adopts full search for both inter and intra prediction. Although there are seven kinds of block size $(16 \times 16,16 \times 8,8 \times 16,8 \times 8,8 \times 4,4 \times 8,4 \times 4)$ for motion compensation, the sum of absolute difference (SAD) of a $4 \times 4$-block can be reused for the SAD calculation of a larger block. Thus, variable block size motion estimation (ME) does not lead to much increase in computation. Intra prediction allows 4 modes for $16 \times 16$-blocks and 9 modes for $4 \times 4$-blocks. The computational load can be estimated as the SAD calculation of thirteen 16x16-blocks and extra operations for interpolation, which are quite small compared to inter prediction. As for the multiple reference frames ME, it contributes to the heaviest computational load. The required operations are proportional to the number of searched frames. Nevertheless, the decrease of prediction residues depends on the nature

\footnotetext{
* The author thanks SiS Education Foundation for financial support.
}
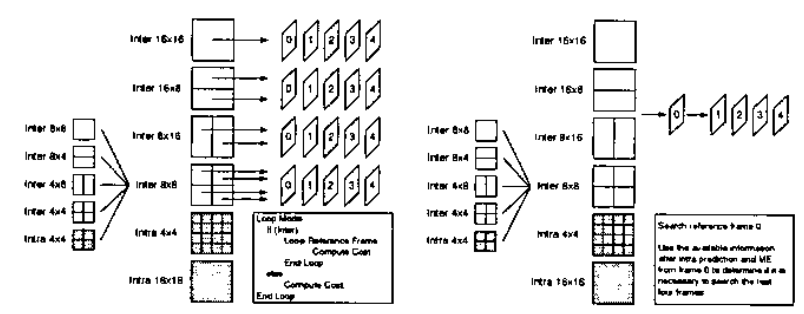

Fig. 1. Searching steps of intra/inter prediction with multiple reference frames in H.264 reference software and our method.

of sequences. Sometimes the prediction gain is very significant, but sometimes a lot of computation is wasted without any benefits. In this paper, we present an effective method to accelerate the multiple reference frames ME without significant loss of video quality. The rest of this paper is organized as follows. in Section 2, we will analyze the statistics of selected mode, residues, and motion vectors for multiple reference frames. In Section 3, we will describe our fast algorithm. Simulation results will be shown in Section 4 . Finally, Section 5 gives a conclusion.

\section{ANALYSIS}

The left side of Fig. 1 shows the searching steps in H.264 reference software. The prediction of a macroblock (MB) is performed mode by mode with full search scheme. The allowed modes are inter $16 \times 16$, inter $16 \times 8$, inter $8 \times 16$, inter $8 \times 8$, intra $4 \times 4$, and intra $16 \times 16$. Note that the inter $8 \times 8$ mode can be further partitioned into smaller blocks. Given an inter-mode, the reference software carries out the matching process reference frame by reference frame. The best mode is chosen by minimizing a Lagrangian cost function, which considers both 2-D 4x4 Hadamard transformed SAD (SATD) and number of bits required to code the side information. The right side of Fig. 1 illustrates our method. In Table 1, we can see that $80 \%$ of the optimal motion vectors (MVs) determined by the reference software belong to the nearest reference frame. Therefore, we first adopt exhaustive search for intra-modes and inter-modes from previous frame. Next, we analyze the available information including selected mode, intra prediction residues, inter prediction residues, and $\mathrm{MVs}$, to determine if it is helpful to search more frames. Intuitively, the prediction gain of multiple reference frames mainly results from occluded and uncovered objects. 
Table 1. Statistics of Reference Frames.

\begin{tabular}{lcc}
\hline Sequences & Previous Frame & Others \\
\hline Coastguard & $75 \%$ & $25 \%$ \\
Container & $91 \%$ & $09 \%$ \\
Foreman & $76 \%$ & $24 \%$ \\
Hall Monitor & $92 \%$ & $08 \%$ \\
Mobile Calendar & $36 \%$ & $64 \%$ \\
Mother and Daughter & $92 \%$ & $08 \%$ \\
Silent & $91 \%$ & $09 \%$ \\
Stefan & $65 \%$ & $35 \%$ \\
Table Tennis & $87 \%$ & $13 \%$ \\
Weather & $90 \%$ & $10 \%$ \\
\hline \hline Average & $80 \%$ & $20 \%$ \\
\hline \multicolumn{2}{l}{ CIF size, search range $|-16,+16| .5$ reference frames, QP $=30}$.
\end{tabular}

We first treat the saving of computation for multiple reference frames in the view point of compression. After prediction, residues are transformed, quantized, and then entropy coded. If we can detect that the transformed and quantized coefficients are very close to zero in the first reference frame, we can turn off the matching process from the rest frames since more computation will not cause any reduction in prediction errors. This concept is very simple and effective. Moreover, DCT, Q, IQ, and IDCT can also be saved by early detection of all-zero quantized coefficients. The quantization steps of $4 \times 4$-residues are described in the following equations:

$$
\begin{aligned}
& q p_{\_} p e r=Q P / 6 \\
& q p_{-} r e m=Q P \% 6 \\
& q p \_ \text {bits }=q p_{-} \text {per }+15 \\
& q p_{-} \text {const }=\left(1<<q_{-} \text {bits }\right) / 6 \\
& Q M[i][j]=\left(|T R[i][j]| \times q u a n t_{-c o e f}\left[q p_{-} \text {rem }\right][i][j]\right. \\
& \left.+q p_{-} \text {const }\right)>>q_{-} \text {bits }
\end{aligned}
$$

where $Q P$ is quantization parameter $(0-51), Q M$ is $4 \times 4$-quantized magnitude, $T R$ is $4 \times 4$-transformed residues, and quant_coef is a 3-D $6 \times 4 \times 4$-matrix. If inequality (6) holds,

$$
|T R|<\left(2^{q-b i t s}-q p_{\text {_const }}\right) / \text { quant_coef } \equiv f(Q P)
$$

the quantized magnitude will be zero, which means the threshold becomes a function of $Q P$ and can be implemented as a lookup table. Besides, $T R$ is not available before transformation, so we assume residues are Laplacian distributed and find the relation between SAD or SATD and $T R$. The threshold is directly applied on SAD or SATD. The detailed derivation is omitted in this paper.

The mode decision result after intra prediction and $\mathrm{ME}$ from previous frame is also a very important cue. In Table $2, \mathrm{~A} \mid \mathrm{B}$ is defined as follows. A is the percentage of a mode after intra prediction and ME from previous frame. $B$ is the percentage of $A$ that optimal reference frame and mode remain unchanged after 5 frames are searched. We can see that $73 \%, 4 \%, 4 \%, 17 \%$, and $2 \%$ of the MBs are selected as $16 \times 16,16 \times 8,8 \times 16,8 \times 8$, and intra, respectively, when only previous one frame is searched. After the rest 4 frames are searched, $90 \%$ of the $16 \times 16-\mathrm{MBs}$ still remain as the optimal selection. As for $16 \times 8-\mathrm{MBs}, 8 \times 16-\mathrm{MBs}, 8 \times 8$ MBs, and intra MBs, the percentages that the optimal mode and reference frame do not change are $65 \%, 65 \%, 34 \%$, and $7 \%$, respectively. This means that $73 \% \times 90 \%+4 \% \times 65 \%+4 \% \times 65 \%+$ $17 \% \times 34 \%+2 \% \times 7 \%=76.82 \%$ of MBs need only previous one
Table 2. Statistics of Selected Modes.

\begin{tabular}{lccccc}
\hline Sequences & $16 \times 16$ & $16 \times 8$ & $8 \times 16$ & $8 \times 8$ & Intra \\
\hline \hline Coastguard & $57 \mid 92$ & $06 \mid 81$ & $06 \mid 78$ & $30 \mid 39$ & $01 \mid 08$ \\
Container & $92 \mid 95$ & $01 \mid 66$ & $01 \mid 61$ & $04 \mid 20$ & $02 \mid 02$ \\
Foreman & $64 \mid 88$ & $08 \mid 68$ & $08 \mid 65$ & $17 \mid 35$ & $03 \mid 06$ \\
Hall Monitor & $90 \mid 98$ & $01 \mid 53$ & $01 \mid 61$ & $07 \mid 44$ & $01 \mid 14$ \\
Mobile Calendar & $49 \mid 50$ & $06 \mid 28$ & $07 \mid 23$ & $37 \mid 20$ & $01 \mid 11$ \\
Mother and Daughter & $89 \mid 97$ & $03 \mid 77$ & $03 \mid 76$ & $04 \mid 27$ & $01 \mid 04$ \\
Silent & $83 \mid 98$ & $03 \mid 78$ & $03 \mid 79$ & $10 \mid 37$ & $01 \mid 12$ \\
Stefan & $47 \mid 84$ & $06 \mid 63$ & $05 \mid 63$ & $38 \mid 39$ & $04 \mid 07$ \\
Table Tennis & $76 \mid 97$ & $04 \mid 71$ & $04 \mid 73$ & $13 \mid 41$ & $03 \mid 08$ \\
Weather & $87 \mid 98$ & $01 \mid 65$ & $02 \mid 64$ & $09 \mid 37$ & $01 \mid 02$ \\
\hline \hline Average & $73 \mid 90$ & $04 \mid 65$ & $04 \mid 65$ & $17 \mid 34$ & $02 \mid 07$ \\
\hline CIF size, search range [-16, +16], QP=30. \\
A|B is defined as follows. \\
A:\% of MBs when only prediction from previous frame is allowed. \\
B: \% of A keeping the same mode and ref. frame after 5 frames are searched.
\end{tabular}

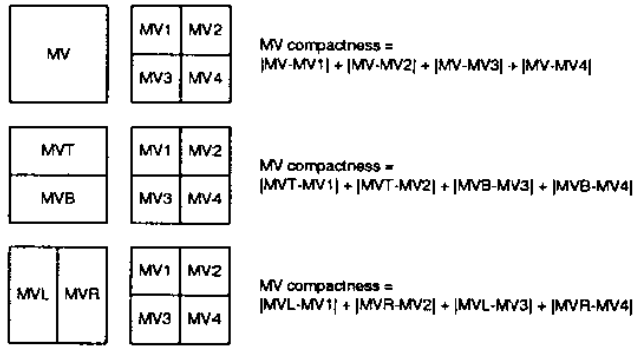

Fig. 2. Definition of MV compactness of a MB.

reference frame, which is quite consistent with the results in Table 1. Furthermore, when a MB is split into smaller blocks for motion compensation using only previous one frame, it means that the motion is discontinuous. In this case, the MB may cross the object boundaries, where occlusion and uncovering often occur. Thus, there is a greater possibility that the best matched candidates belong to the other 4 reference frames. When the intra-mode has better prediction than inter-modes from previous frame, the MB may belong to uncovered parts or new objects. The best candidates are very likely to be found in other 4 reference frames, too.

Now we try to find the correlation between MV distribution and optimal reference frames. After ME from previous one frame, we have one MV for $16 \times 16-\mathrm{MB}$, two MVs for $16 \times 8-\mathrm{MB}$, two $\mathrm{MVs}$ for $8 \times 16-\mathrm{MB}$, and four MVs for $8 \times 8-\mathrm{MB}$. If the best mode is $16 \times 16,16 \times 8$, or $8 \times 16$, the definition of MV compactness for each of the 3 modes is shown in Fig. 2, respectively. Next, we keep on searching the other 4 frames. If the optimal frame or mode of a MB does not change after ME from 5 frames, we classify these MBs as type I. Otherwise, we classify them as type II. The average MV compactness of type I and type II for each sequence is shown in Table 3. The MV compactness of MBs with optimal reference frame belonging to the rest 4 frames tends to be larger than that of MBs predicted by previous frame. Therefore, if the MV compactness of a MB after ME from previous frame is very small, we should stop searching the rest 4 frames.

The texture is also taken into consideration. The reduction of residues by applying multiple reference frames is more significant 
Table 3. Statistics of Average MV Compactness.

\begin{tabular}{lccc}
\hline Sequences & \multicolumn{1}{c}{$16 \times 16$} & $16 \times 8$ & $8 \times 16$ \\
\hline Coastguard & $08.9 \mid 12.6$ & $09.9 \mid 12.5$ & $07.8 \mid 09.5$ \\
Container & $07.4 \mid 12.3$ & $07.7 \mid 09.2$ & $07.1 \mid 08.9$ \\
Foreman & $08.3 \mid 16.4$ & $10.1 \mid 13.2$ & $10.5 \mid 13.2$ \\
Hall Monitor & $08.4 \mid 14.5$ & $09.8 \mid 12.7$ & $10.7 \mid 12.5$ \\
Mobile Calendar & $07.1 \mid 11.8$ & $07.4 \mid 11.2$ & $09.5 \mid 10.9$ \\
Mother and Daughter & $06.9 \mid 10.5$ & $07.7 \mid 11.4$ & $10.0 \mid 11.6$ \\
Silent & $07.2 \mid 11.9$ & $10.4 \mid 14.2$ & $11.6 \mid 16.2$ \\
Stefan & $08.4 \mid 22.4$ & $11.8 \mid 16.5$ & $12.7 \mid 17.3$ \\
Table Tennis & $08.6 \mid 17.5$ & $08.1 \mid 13.4$ & $10.4 \mid 16.7$ \\
Weather & $07.7 \mid 11.8$ & $07.1 \mid 08.5$ & $07.2 \mid 10.7$ \\
\hline \hline Average & $07.9 \mid 14.2$ & $09.0 \mid 12.3$ & $09.8 \mid 12.8$ \\
\hline \multicolumn{4}{l}{ CiF size, search range [-16, +16]. QP=30. } \\
The unit of MV compactness is quarter pixel. \\
1|Il is defined as follows. \\
1: optimal ref. frame and mode do not change after ME from 5 frames. \\
11: optimal ref. frame and mode change after ME from 5 frames.
\end{tabular}

at object boundaries, where occlusion and uncovering often occur. The texture of object boundaries should be more complex than other flat regions. We use SATD after intra prediction to represent the complexity of texture of a MB. In Table 4, intra prediction and $\mathrm{ME}$ from previous one frame is first applied for each MB. Then we focus on the MBs having the best mode as $16 \times 16,16 \times 8$, and $8 \times 16$. Again, we keep on searching the rest 4 frames and classify the MBs into two types. MBs that do not change optimal reference frame and mode are classified as type P, while MBs with different reference frame and mode are denoted as type $Q$. It is clear that the SATD of intra prediction for type P is smaller than that for type Q. Therefore, if a MB is significantly textured, we should search more frames. However, it is also clear from Table 4 that this threshold value should be adaptive with different scenes or sequences.

There are some exceptions in Table 4, such as $16 \times 8$ and $8 \times 16$ modes for Hall Monitor, $16 \times 8$ mode for Silent. These are due to the complicated texture of stationary background that only requires one reference frame. In these cases, if we do not want to lose video quality, the threshold for SATD of intra prediction should be small enough, which will cause waste of computation for highly. textured stationary background. Fortunately, we can use the MV compactness to prevent these situations. Our algorithm will be described in the next section. Let us summarize the analysis as follows. After intra prediction and ME from previous one frame,

- If $16 \times 16$ mode is selected, the optimal reference frame tend to be unchanged.

- If inter-modes with smaller blocks are selected, searching more frames tend to be helpful.

- If MVs of larger blocks are similar to MVs of smaller blocks, it is likely that no occlusion or uncovering occurs in MB, so one reference frame may be enough.

- If MVs of larger blocks are more different from MVs of smaller blocks, MB often crosses object boundaries and thus requires more reference frames.

- If the texture of a MB is very complicated. it may require more reference frames.
Table 4. Statistics of Average SATD of Intra Prediction.

\begin{tabular}{|c|c|c|c|}
\hline Sequences & $16 \times 16$ & $16 \times 8$ & $8 \times 16$ \\
\hline$\overline{\overline{\text { Coastguard }}}$ & $\begin{array}{l}3599 \\
5168\end{array}$ & 499325587 & $\overline{4846 \mid 5150}$ \\
\hline Container & $3103 \mid 8218$ & $4813 \mid 5242$ & 5960 \\
\hline Foreman & $2104 \mid 3094$ & $3103 \mid 3269$ & 25623290 \\
\hline Hall Monitor & 23413875 & $4015 \mid 2149$ & 41821950 \\
\hline Mobile Calendar & $6165 \mid 8371$ & $6843 \mid 8239$ & $6290 \mid 8723$ \\
\hline Mother and Daughter & $1482 \mid 2472$ & $2488 \mid 2388$ & $2250 \mid 2501$ \\
\hline Silent & $2881 \mid 2904$ & $3010 \mid 2604$ & $3227 \mid 3256$ \\
\hline Stefan & $3723 \mid 5981$ & $5795 \mid 6113$ & $5975 \mid 6188$ \\
\hline Table Tennis & $3025 \mid 3920$ & $3773 \mid 3420$ & $3725 \mid 3827$ \\
\hline Weather & $4648 \mid 4324$ & $3985 \mid 3745$ & $4286 \mid 4669$ \\
\hline$\overline{\text { Average }}$ & 33074833 & $4 2 7 6 \longdiv { 4 2 7 6 }$ & $\begin{array}{l}4330 \\
4556\end{array}$ \\
\hline \multirow{2}{*}{\multicolumn{4}{|c|}{$\begin{array}{l}\text { CIF size, search range }[-16,+16], Q P=30 \text {. } \\
P \mid Q \text { is defined as follows. }\end{array}$}} \\
\hline & & & \\
\hline \multicolumn{4}{|c|}{ P: optimal ref. frame and mode do not change after ME from 5 frames. } \\
\hline
\end{tabular}

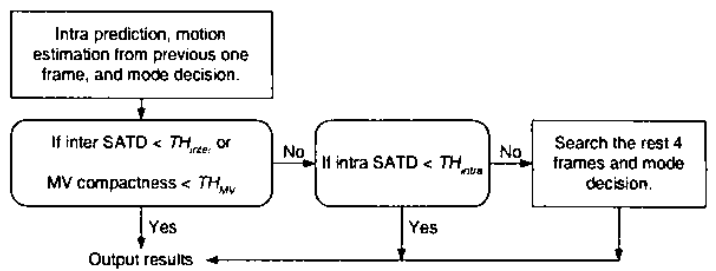

Fig. 3. Fast algorithm for multiple reference frames ME.

\section{PROPOSED ALGORITHM}

According to the above analysis, we propose a fast algorithm for multiple reference frame ME to save the computation of full search and to maintain the same video quality. The steps are shown in Fig. 3. Note that $T H_{\text {inter }}$ is a function of $Q P$ and is implemented as a look-up table. $T H_{M V}$ is empirically obtained. $T H_{\text {intra }}$ must be adaptive with different scenes. Currently, we use the number of intra $\mathrm{MBs}$ in previous coded frame to detect scene change. If more than $10 \%$ of the MBs are intra-coded, we will adjust $T H_{\text {intra }}$. The detailed derivation of $T H_{\text {intra }}$ is omitted due to the limited space. As shown in Fig. 4, we connected ten standard sequences together to show the dynamic adjustment of $T H_{\text {intra }}$ according to the number of intra MBs in previous frame.

\section{SIMULATION RESULTS}

Figure 5 compares the rate distortion curves of the reference software and the proposed algorithm. It is shown that the maximum peak signal to noise ratio (PSNR) drop is $0.2 \mathrm{~dB}$ (Hall Monitor). The average PSNR drop is less than $0.05 \mathrm{~dB}$, so that the two curves for each sequence are hardly distinguishable. Table 5 shows the miss detection rate and the false alarm rate of the proposed algorithm. Note that miss detection of optimal reference frames leads to the degradation of PSNR, and the false alarm results in waste of computation. In fact, $T \boldsymbol{H}_{\text {intra }}$ provides an easy trade-off between speed and quality. Adjusting $T H_{\text {intra }}$ cannot decrease the miss detection rate and false alarm rate at the same time. The higher the $T H_{\text {intra }}$, the more the computation is saved. The lower the 


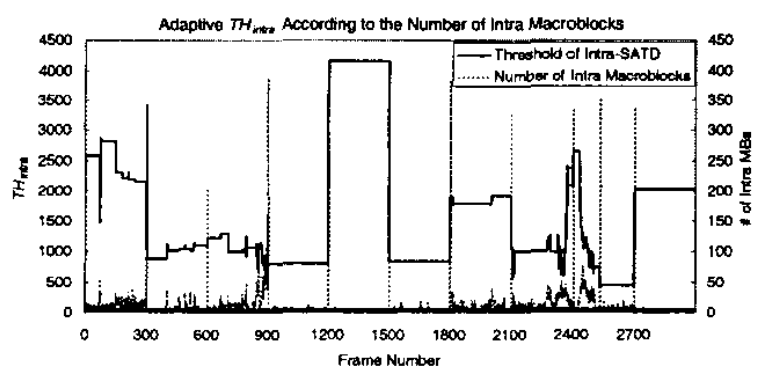

Fig. 4. Adaptive $T H_{\text {intra }}$ according to the number of intra MBs.

Table 5. Miss Detection and False Alarm Rates.

\begin{tabular}{lcc}
\hline Sequences & Miss Detection & False Alarm \\
\hline Coastguard & $6.21 \%$ & $41.15 \%$ \\
Container & $1.90 \%$ & $24.96 \%$ \\
Foreman & $2.57 \%$ & $47.42 \%$ \\
Hall Monitor & $5.37 \%$ & $12.83 \%$ \\
Mobile Calendar & $5.86 \%$ & $31.60 \%$ \\
Mother and Daughter & $2.67 \%$ & $24.57 \%$ \\
Silent & $3.73 \%$ & $19.65 \%$ \\
Stefan & $5.62 \%$ & $42.36 \%$ \\
Table Tennis & $2.41 \%$ & $25.15 \%$ \\
Weather & $2.64 \%$ & $12.30 \%$ \\
\hline Average & $3.90 \%$ & $28.20 \%$ \\
\hline
\end{tabular}

CIF size, search range $[-16,+16]$.

$T H_{\text {intra }}$, the less the PSNR drop is achieved. The average miss detection rate is only $3.90 \%$, which means $96.10 \%$ of MBs can find the optimal reference frame. The average false alarm rate is $28.20 \%$, which means we should further improve our algorithm to save the $28.20 \%$ of computation while keeping the miss detection rate from rising in the mean time. Given a budget of computation resources, how to select $T H_{\text {intra }}$ will also be our future work. Figure 6 shows the number of average searched frames for the reference software and the proposed algorithm. It is shown that $10 \% \cdot 67 \%$ of ME operations can be saved.

\section{CONCLUSION}

We proposed a simple and effective fast algorithm for multiple reference frames motion estimation. We first analyzed the available information after intra prediction and motion estimation from previous one frame. Then we applied several threshold values on the available information to determine if it is necessary to search more frames. Experimental results showed that our method can save $10 \%-67 \%$ of ME computation depending on sequences while keeping the quality nearly the same as full search scheme.

\section{REFERENCES}

[1] Commitlee Draft of Joint Video Specification (ITU-T Rec. H.264 and ISO/IEC 14496-10 AVC), July, 2002.

[2] Joint Video Team (JVT) software JM4.3, October, 2002.
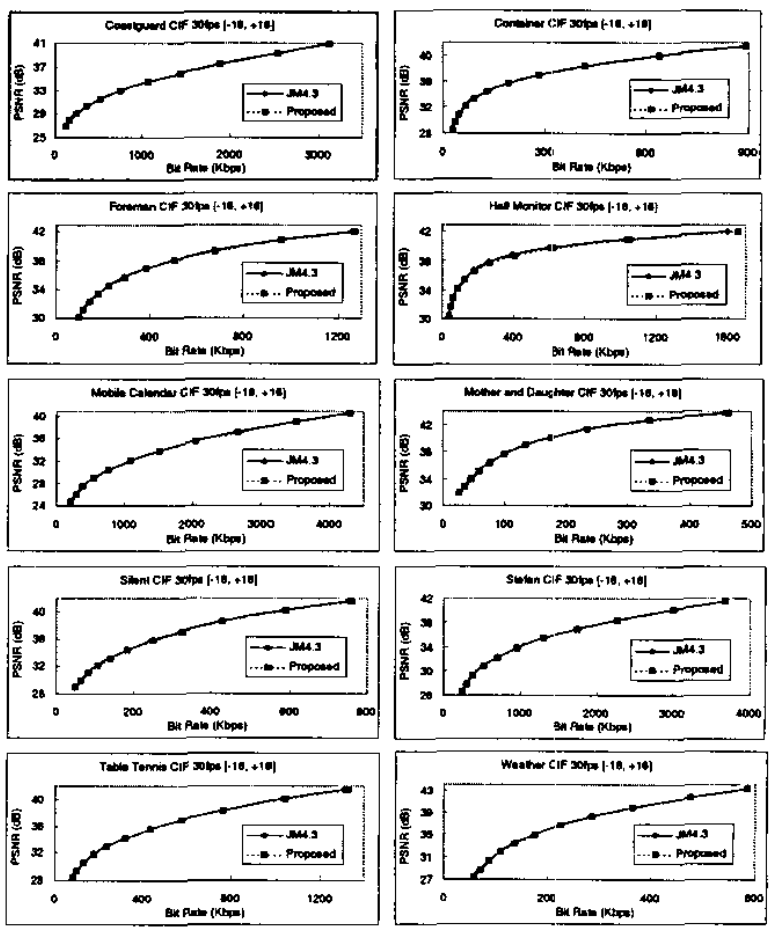

Fig. 5. Rate distortion curves of various sequences.
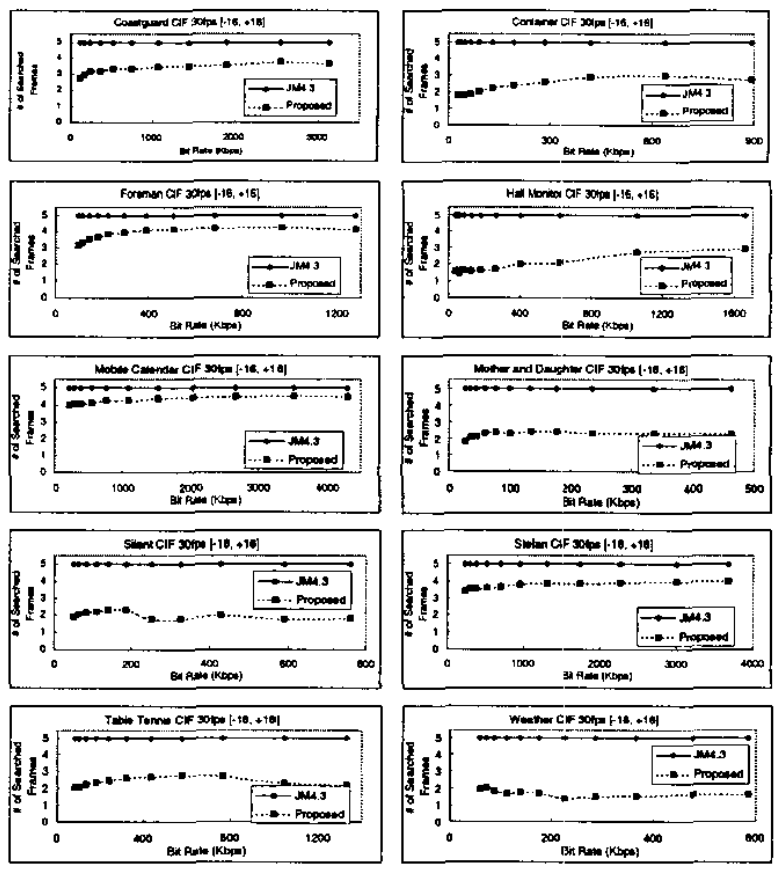

Fig. 6. Average searched frames for various sequences. 